\title{
Newborn blood spot screening and genetic services: A survey of Minnesota primary care physicians
}

Diane B. Thompson, $M S^{1,4}$, Mary J. Ahrens, $M S^{1}$, Bonnie S. LeRoy, MS $S^{1}$, Dana Brown, $M S^{3}$, and Susan A. Berry, MD ${ }^{1,2}$

\begin{abstract}
Purpose: To (1) obtain guidance on the preferred content and format of quick reference newborn blood spot screening information from the Minnesota Department of Health; (2) determine primary care physicians' perceptions of the benefits of genetic services; and (3) determine primary care physicians' satisfaction with genetic counseling services. Methods: A written survey was mailed to family physicians and pediatricians in Minnesota ( $n$ $=300$ ). Results: Eighty physicians responded ( $28 \%$ response rate). Whereas $70 \%$ of respondents felt previous information received from the newborn screening program was adequate, $83 \%$ were interested in quick reference information. The majority of physicians preferred this information as a laminated sheet (63\%). Physician procedure for an abnormal screen, newborn screening program protocol for an abnormal screen, and disease treatment and follow-up information were recommended for inclusion on quick reference. Over half of physicians agreed with the following benefits of genetic services: provide testing options (88\%); evaluate family members (88\%); reduce parental anxiety (87\%); provide resources (83\%); provide diagnostic information (76\%); determine medical needs (67\%); and determine emotional needs (51\%). Ninety-nine percent of physicians were satisfied with genetic counseling services. Conclusions: Physicians indicated that reference material for primary care physicians should include a quick reference card with specific categories of information. Newborn screening programs should attempt to increase physician awareness of genetic services, including the subsequent medical and psychosocial benefits for their patients. Genet Med 2005:7(8):564-570.
\end{abstract}

Key Words: newborn blood spot screening, genetic counseling, primary care physicians, genetic services, tandem mass spectrometry

\section{INTRODUCTION}

Primary care providers are critical for effective communication of results from newborn blood spot screening to parents, in addition to providing parental guidance and education. ${ }^{1}$ The suggested role of the primary care physician is to "assist the family in understanding the diagnosis, symptoms, and potential implications of the condition, as well as the availability if genetic counseling, family testing, and other family support services". ${ }^{2}$ Because of this central role, improved physician awareness and education about newborn blood spot screening are vital to the efficient functioning of these programs. ${ }^{1}$ The Minnesota Department of Health (MDH) has developed educational material for physicians and parents that is available on their web site. ${ }^{3}$ However, there was little known about the cur-

From the ${ }^{I}$ Graduate Program in Genetic Counseling, University of Minnesota, Minneapolis, Minnesota, ${ }^{2}$ Department of Pediatrics and Institute of Human Genetics, University of Minnesota, Minneapolis, Minnesota; ${ }^{3}$ Newborn Screening Program, Minnesota Department of Health, Minneapolis, Minnesota; and ${ }^{4}$ the Department of Obstetrics and Gynecology, Mercy Medical Center, Baltimore, Maryland.

Susan Berry, MD, Department of Pediatrics, 4-150 MT, 515 Delaware St. SE, Minneapolis, Minnesota, 55455.

Received: May 31, 2005

Accepted: June 9, 2005

DOI: 10.1097/01.GIM.0000177417.61006.a6 rent awareness and knowledge about the newborn blood spot screening by physicians. In addition, there was no data about physician satisfaction with educational material provided by the MDH. We undertook a survey of Minnesota health care providers to assess these issues, as well as physicians' understanding of genetic services and their satisfaction with genetic counseling for their patients, some of whom had abnormal blood spot screens.

Newborn blood spot screening is a public health program that provides early identification and treatment for medical conditions that can lead to decreased quality of life, disability, or death. Although newborn blood spot screening occurs nationally, the specific diseases included in the screening vary between states. Until 2001, Minnesota newborns were screened for 5 conditions: phenylketonuria (PKU), galactosemia, hemoglobinopathies, congenital adrenal hyperplasia, and congenital hypothyroidism. Minnesota began using tandem mass spectrometry (MS/MS) for newborn blood spot screening as part of a pilot study in July of 2001 and added the diseases detectable by this technology to the newborn blood spot screening panel in 2003.

With the addition of MS/MS technology for newborn blood spot screening, additional disorders of amino acid metabolism, organic acidemias, and fatty acid oxidation are detectable. With a large number of additional conditions ascertained, each with varying symptoms, complications, and care plans, the 
clinical implications of MS/MS technology have been confusing for primary care physicians. In 2002, 67,839 babies were screened in Minnesota via traditional and MS/MS newborn blood spot screening, with 1,077 presumptive positive screens for one of the tested conditions (unpublished data, 2003). Because MS/MS technology increases the number of detectable diseases, health care providers will need additional information as they provide follow-up care to newborns with a positive screen. ${ }^{4}$ There is general agreement that families and health care providers need information about newborn blood spot screening. ${ }^{5-10}$ Korson suggested that pediatricians need to know the types of metabolic diseases included in MS/MS technology, along with their clinical presentation. ${ }^{6} \mathrm{He}$ also outlined a protocol in the event of an abnormal result that included consulting a metabolic specialist regarding medical ramifications and facilitating referral for diagnostic evaluation. Other responsibilities beyond these could include contacting the family, checking the infant's health, and sending confirmatory information to the newborn screening program. However, no published studies asked primary care providers for newborns what type of information they would like provided to them. When developing educational materials, the use of models developed by other states may be valuable. In a survey of communication practices between newborn screening programs and primary care physicians, $86 \%$ of states provided professional education and training on newborn blood spot screening. ${ }^{7}$ Details regarding this education and training were not specified. Sixty nine percent of states targeted the primary care physician, and $80 \%$ of all states have designated health care professional responsibilities for follow-up in newborn blood spot screening. ${ }^{7}$ Formats used by state agencies included practitioner manuals, physician newsletters, and web sites that supply physician information.

With the expansion of newborn blood spot screening, more infants with genetic conditions will be identified. A recent estimate is a $50 \%$ to $100 \%$ increase in identified patients each year. ${ }^{11}$ Therefore, the demand for medical follow-up, including genetic counseling, will increase. Newborn blood spot screening expansion presents a critical time to assess the satisfaction and effectiveness of genetic services so that improvement in the provision of these services can take place before attempts are made to meet increased demand. Although studies show genetic counseling is effective for providing genetic information, few studies assess patient satisfaction of services provided by genetic counselors. ${ }^{12}$ Current studies focus on educational and reproductive outcome in patients to measure genetic counseling success. ${ }^{13}$ The primary care physician may be a good assessor of parental counseling satisfaction due to their role of managing the newborn and amount of parent contact. Physician satisfaction may also influence use of and referral to genetic services. With the assistance of $\mathrm{MDH}$, primary care physicians were surveyed to determine how the Minnesota newborn screening program could best develop informational materials for physicians. We sought to (1) obtain physicians' opinions on the preferred content and format of newborn blood spot screening information from MDH; (2) determine the primary care physicians' understanding of the role of genetic counseling; and (3) measure experience in their practice with genetic counseling services.

\section{MATERIALS AND METHODS}

A mailing list of 300 physicians was randomly selected from a list of 1600 Minnesota physicians who see newborn patients. The demographics of the source list were maintained for location of practice: Minneapolis/St. Paul metropolitan area (metro) and nonmetro for both family practitioners and pediatricians. The number of clinicians in each category reflected state distributions. The metro area was identified as the seven county area surrounding Minneapolis and St. Paul. Based on these criteria, 51 surveys were sent to Metro Pediatricians, 108 were sent to Metro Family Physicians, 18 were sent to Nonmetro Pediatricians, and 123 were sent to Nonmetro Family Physicians. A total of 80 surveys out of 300 were completed and returned ( $28 \%$ response rate). There is a $90 \%$ level of confidence that this size sample is statistically representative of the surveyed physician population. ${ }^{14}$ Metro pediatricians returned 19 surveys (37\% response); 21 Metro family physicians returned the survey (19\% response); 6 surveys were returned by nonmetro pediatricians (33\% response); 32 of the nonmetro family physicians returned the survey (26\% response).

A written survey with 19 questions was used with approval from the University of Minnesota Institutional Review Board (see Appendix). This survey consisted of multiple choice and short answer questions. Areas of the survey included the following: past information received from $\mathrm{MDH}$; future information to be developed by MDH; knowledge of state public programs that assist children with special needs; genetic services; genetic counseling; and demographics. Leikert type scales were used for two questions. Descriptive statistics were calculated for responses to each survey question. Groupings of years of practice were determined by standard deviations from the average of the respondent sample. Confidence intervals were determined using Rweb statistical software ${ }^{15}$ and functions for each survey question, based on the entire respondent pool. Two-tailed $t$ tests were conducted to examine demographic differences and determine statistically significant relationships between selected variables. Because some surveys were returned partially completed, each question is based on the number of respondents for that question. Respondents were encouraged to check all answers that apply, so total percentages when all answers for each question are combined can be $>100 \%$.

\section{RESULTS}

Demographic characteristics of the 80 responding primary care physicians are presented in Table 1 . The majority of respondents were family physicians $(66 \%)$ and most respondents were located in the metro area of Minnesota (51\%). The average number of years in practice was 13.7 years, ranging from 2.5 years to 38 years. The majority of respondents re- 
Table 1

Respondent demographics $(n=80)$

\begin{tabular}{lcc}
\hline & $\mathrm{n}$ & $\%$ \\
\hline Area of medical expertise & & \\
Family practice & 53 & 66.25 \\
Pediatrics & 25 & 31.25 \\
Location of practice & & \\
Metro & 41 & 51.25 \\
Nonmetro & 38 & 47.5 \\
Years of practice & & \\
$2.5-5$ & 14 & 17.5 \\
$5.5-12$ & 28 & 35 \\
$13-21$ & 24 & 30 \\
$22-30$ & 11 & 13.75 \\
$31-38$ & 2 & 2.5 \\
Number of positive results in last 5 years & & \\
0 & 21 & 26.25 \\
$1-3$ & 39 & 48.75 \\
$4-7$ & 7 & 8.75 \\
8 or More & 10 & 12.5 \\
\hline
\end{tabular}

ported receiving one to three positive newborn blood spot screen results in the last 5 years (49\%). Twenty six percent had not had any positive newborn blood spot screening results in the last 5 years. Most respondents had received normal newborn blood spot screening results (95\%, 71 of 75$)$ and a majority had received abnormal screening results (75\%, 56 of 75$)$. Thirty nine percent of respondents (29 of 75) reported receiving information on specialists available for patient referral after abnormal screens, and 28\% (21 of 75) received information specific to the diagnosis indicated by an abnormal screen. Only one respondent (1\%) reported using the $\mathrm{MDH}$ web site to access any information.

A majority of respondents reported that information received from the MDH newborn screening program was adequate $(70 \%, 54$ of 77$) .29 \%$ of respondents (22 of 77) felt the information was not enough, and only one physician ( $1 \%, \mathrm{CI}$ $0.03-7.0)$ regarded it as more than adequate. When questioned as to whether physicians would want quick reference information on newborn blood spot screening, a majority were interested (83\%, CI 72.5-90.6\%). A majority also expressed interest in receiving quick reference information in a laminated sheet format $(63 \%$, CI $50-74 \%)$. The second most-preferred format was via the MDH newborn screening web site (38\%, CI 26$50 \%)$. Physicians also indicated interest in a pocket reference information sheet $(31 \%$, CI $20-44 \%)$. An equal number of respondents were interested in a PDA download format and a brochure $(22 \%$, CI $13-33 \%)$. A minority of physicians preferred the information to be presented via a letter (14\%, CI 7-25\%), newsletter, manual, fax (each 9\%, CI 4-19\%), or tele- phone call (5\%, CI 1-13\%). Pediatricians and family practitioners differed significantly on the format preferences $(P=$ $0.045)$. They preferred a laminated information sheet $(54 \%$ and $65 \%$, respectively) or web site $(45 \%$ and $35 \%$, respectively), but did not agree on the remaining formats.

Respondents rated several types of information on a scale of one to five based on how important it is to be included in quick reference material (Table 2). The highest rated information was practitioner follow-up procedure for an abnormal screen result (4.47, SD 0.80). The next highest rated information includes the newborn blood spot screening protocol for an abnormal screening result (4.45, SD 0.79), and disease treatment options and follow-up (4.03, SD 1.15).

A number of benefits of genetic services were proposed to physicians (Table 3). Respondents agreed most often that genetic services can help patients in the following ways: by providing the family with information about testing options (88\%); evaluation and counseling of family members (88\%); reduction of parental anxiety (87\%); and providing resources specific to 1.8 the diagnosis and prognosis of an affected individual.

Physicians who have referred to genetic counseling services were asked about their satisfaction with these services. Ninety nine percent of these respondents reported that they were satisfied (68 of 69). Only one respondent was not satisfied (1\%, CI $0.03-7.8 \%)$. When presented with specific reasons for satisfaction and dissatisfaction, $98 \%$ of physicians agreed that genetic counselors provide helpful genetic information to the family (see Table 4). Three individuals expressed their own reasons for dissatisfaction: lack of information back to provider, genetic counselor is not easily available, and quantity of information provided by the genetic counselor overwhelms patients.

Table 2

Information to be included for quick reference $(n=80)$

Mean

n $\quad \%$ Importance $^{a} \quad \mathrm{SD}$

\begin{tabular}{|c|c|c|}
\hline Practitioner procedure - abnormal screen & 7897.5 & 4.47 \\
\hline
\end{tabular}

$\begin{array}{lllll}\text { Newborn screening protocol - abnormal screen } & 75 & 93.8 & 4.45 & 0.79\end{array}$

$\begin{array}{lllll}\text { Disease treatment and follow-up } & 76 & 95 & 4.03 & 1.15\end{array}$

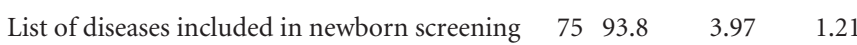

$\begin{array}{lllll}\text { Contact information - specialists } & 79 & 98.8 & 3.78 & 1.11\end{array}$

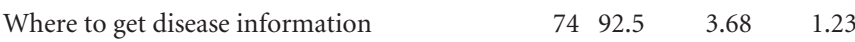

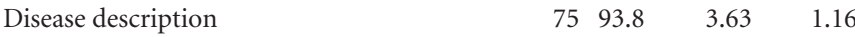

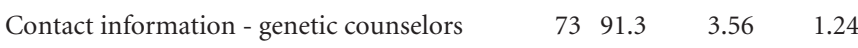

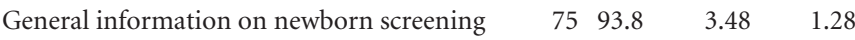

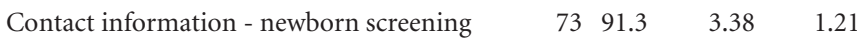

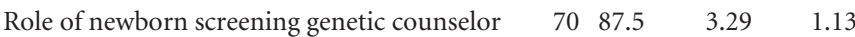

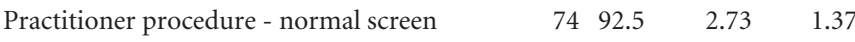

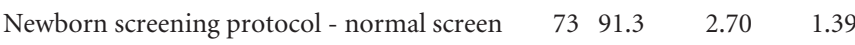

${ }^{a}$ Based on a 5 -point scale, where $1=$ not at all important, $3=$ important, $5=$ very important 
Table 3

Benefits of genetic services $(n=76)$

\begin{tabular}{llll}
\hline & $\mathrm{n}$ & $\%$ & $\mathrm{CI}(\%)$ \\
\hline Provide testing options & 67 & 88 & $79-94.4$ \\
Evaluation of family members & 67 & 88 & $79-94.4$ \\
Reduce parental anxiety & 66 & 87 & $77-93.5$ \\
Provide resources & 63 & 83 & $73-91$ \\
Provide information about diagnosis & 58 & 76 & $65-85$ \\
Determine medical needs & 51 & 67 & $55-77$ \\
Determine emotional needs & 39 & 51 & $40-63$ \\
\hline
\end{tabular}

Table 4

Reasons for satisfaction or dissatisfaction with genetic counseling $(n=65)$

\begin{tabular}{lccc}
\hline & $\mathrm{n}$ & $\%$ & $\mathrm{CI}(\%)$ \\
\hline Satisfaction & & & \\
$\quad$ Provided information to family & 64 & 98 & $91.72-99.96$ \\
$\quad$ Assisted family with genetic needs & 54 & 83 & $71.73-91.24$ \\
$\quad$ Provided information to referring physician & 49 & 75 & $63.13-85.23$ \\
Dissatisfaction & & & \\
$\quad$ Not easily available & 1 & 2 & $0.04-8.28$ \\
$\quad$ Overwhelmed patients & 1 & 2 & $0.04-8.28$ \\
$\quad$ Lack of information to referring physician & 1 & 2 & $0.04-8.28$ \\
\hline
\end{tabular}

None of the respondents agreed with the following proposed reasons for dissatisfaction: not enough benefit for cost of visit, confused the patient, or did not add to patient care.

The majority of physicians reported that they have referred patients to genetic counselors. Thirty- four percent of respondents have referred one to two times (26 of 77), and 31\% have referred three to five times ( 24 of 77 ). Twenty-two percent referred five times or more ( 17 of 77 ), and 13\% of physicians have never referred patients to a genetic counselor (10 of 77). Physicians who have not had patients with positive newborn blood spot screening results referred to genetic counseling significantly less often than those physicians with patients who have had positive newborn blood spot screening results $(P=$ 0.007). Thirty-three percent of newborn blood spot screening inexperienced physicians have never referred to a genetic counselor, as compared to $6 \%$ of newborn blood spot screening experienced physicians. Nine of the 10 respondents who reported never referring to genetic counseling expressed their reasons for not referring. Five physicians did not need genetic counseling services, three were not familiar with genetic counseling, and two reported not having a genetic counselor in the area.

\section{DISCUSSION}

The results of this survey uncovered current flaws in the reporting practices of newborn blood spot screening programs and demonstrated that physicians have interest in receiving additional material from the newborn screening program in formats other than have been previously utilized. There were $5 \%$ of physicians who did not report receiving normal results and may be experiencing a problem of indirect communication, which is common nationally. The MDH newborn screening program at the time of this survey did not report newborn blood spot screening results directly to the primary care physician, but to the birth hospital. The birth hospital then had the responsibility of contacting the primary care physician. In a national survey of pediatricians, $26 \%$ did not receive normal newborn blood spot screening results. Barriers to primary care physicians in receiving newborn blood spot screening results include the following: physicians not having privileges at the birth hospital, transfer of medical care to new physician, babies born in other states, clinic personnel time to track newborn blood spot screening results, infant name change, or indirect communication between the newborn screening program and the primary care physician.

Current newborn blood spot screening information forwarded by the newborn blood spot screening department is not getting to the primary care physician. The $\mathrm{MDH}$ routinely faxes the name and contact information of a condition-specific specialist and diagnostic information with a positive newborn blood spot screening result. However, only $39 \%$ of physicians reported receiving specialist referral information and $28 \%$ received information specific to a diagnosis. Because $75 \%$ of physicians have received abnormal screening results, these numbers should be higher. It is possible that the physician did not see this material if other clinic employees are responsible for the incorporation of this information into a patient's chart, or physicians may not remember receiving this information.

Physicians are interested in new information from the newborn blood spot screening program. Overall, physicians felt the previously received material from the newborn screening program was adequate $(70 \%)$. We inferred from this that there is room for improvement. The overwhelming interest in new material ( $83 \%$ of physicians) confirms this need. Physicians may be anticipating future increases in complexity of newborn blood spot screening and therefore are indicating a need for additional supportive material. Other studies have also witnessed the need for health provider educational material about the implications of newborn blood spot screening results. ${ }^{9}$ The recent ACMG Newborn blood spot screening Expert Group "identified a clear gap in the information available and information needed by primary care professionals to facilitate an immediate response in the event of a screen-positive infant." 10

Surveyed physicians preferred receiving quick reference information as a laminated information sheet $(63 \%)$ or through a web site $(38 \%)$, which are different from current and suggested formats. The current MDH information including specific specialist referral is provided initially by phone and then faxed and mailed on multiple loose conventional pages. Similarly, the ACMG Newborn blood spot screening Expert Group developed Action Sheets for each condition, which are presented as loose paper sheets. ${ }^{10}$ The interest in an Internet-based 
format is contradictory to the current usage of the MDH newborn screening program web site that contained extensive information for parents and practitioners. This discrepancy may be indicative of the physicians' lack of awareness of the web site. Other factors of usage may be the type of information on the web site, where they need the information (in a clinic room vs. office), or how immediately they need the information. The $\mathrm{MDH}$ newborn screening program web site URL is currently included on the written disease description sent to physicians at the time of an abnormal result, but may need to made be more visible to physicians. The surveyed clinicians indicated that the most preferred information to include is the practitioner procedure for abnormal screen results; physicians have been previously advised to establish a procedure for abnormal results in their own practice. It may be that physicians rarely get a positive screen result, and therefore have not developed a standard procedure. Also, most of the suggested information to include on quick reference was deemed important. However, the reality of including all the requested information on one laminated reference sheet may be difficult. It may be most feasible to have a general practitioner procedure for abnormal screening results along with contact numbers or a web site for more specific information. The information that physicians requested in this study, except for a complete list of diseases screened, is included in the current information sent to physicians when there is an abnormal screen. The current MDH newborn blood spot screening reports include the following: a listing of results by disease or disease category (galactosemia, hemoglobinopathies, hypothyroidism, congenital adrenal hyperplasia, amino acidemias, fatty acid oxidation disorders, and organic acidemias); a recommendation for follow-up and specialist referral; and a fact sheet on the disease in question. This fact sheet includes a definition of the disease, incidence, inheritance, characteristics, brief newborn screening program protocol and practitioner procedure for testing validation, management and outcome of disease, practitioner management of babies with unconfirmed positive results, and the method used for testing. This is similar in content to what is included in the ACMG proposed Action Sheets, except the Action Sheets include references for additional information such as Gene Tests. ${ }^{10}$ However, one physician commented that clinical implications or management recommendations were not included with the abnormal results, and the specialists were always in the metro area. Another physician reported that they were asked by another physician about follow-up procedure and recommended to include a web site and information sheet with a positive test result. These responses suggest that the current report sent by the newborn screening program is either not getting to or read in its entirety by the physician, or that the information is confusing. New measures are needed to ensure that physicians receive the information already available that meet these surveyed preferences.

Over $50 \%$ of physicians agreed with all proposed benefits of genetic services, which may indicate their knowledge of these services. However, physicians may have agreed with these benefits because they sounded accurate. Up to $88 \%$ of them agreed with one of these benefits, which are current responsibilities of medical geneticists, genetic counselors, nurses, and dietitians. Physicians proposed no other benefits. Other specific responsibilities of genetic counseling are as follows: to determine parental carrier risk and offer testing ${ }^{16}$; to supply counseling after false positive results or for heterozygous infants ${ }^{17}$; and to provide information about the prognosis for asymptomatic but affected babies. ${ }^{8}$ This last responsibility is especially crucial for the expansion of newborn blood spot screening because the prognosis and outcome for identified babies may not be well defined.

There is clearly a need to increase physician understanding of genetic services. Over $40 \%$ of physicians did not recognize some of these benefits, and only $51 \%$ agreed that genetic services identify social and emotional needs. Psychosocial support is one of the responsibilities and goals of genetic counseling, ${ }^{18,19}$ Also, five of ten nonreferring physicians reported that genetic counseling services were not needed. No further information was provided as to their reasons for not needing services. One of these physicians did not respond to the possible (benefits of genetic services. This may be interpreted as not understanding the benefits of genetic services, or that genetic services are not important. The other four physicians agreed that there are benefits to genetic services, so it may be that they have not identified an indication to refer patients. Physicians and their patients may benefit from further education and awareness of the role of genetic services. An estimated 3\% to $5 \%$ of babies are born with some birth defect or condition associated with genetic factors, $10 \%$ of school-aged children have sensory, developmental, behavioral or emotional difficulties that may have a genetic component, and all families are suspected to have at least one genetic disorder that could necessitate a genetic referral. ${ }^{21}$ The National Newborn Screening and Genetics Resource Center suggested that state departments of health educate primary care providers about genetic services and when to refer their patients to genetic practitioners. ${ }^{20}$ Therefore, newborn screening programs may also wish to educate physicians about genetic services to increase awareness and subsequent medical benefits for their patients.

Of the 10 physicians who reported no referrals to a genetic counselor in the last 5 years, three were not familiar with genetic counseling services: they were metro physicians who had not had babies with a positive result in the last 5 years. Their experience as practitioners varied from 4 to 22 years. Also, physicians who have not had babies with a positive newborn screening result referred to a genetic counselor significantly less frequently than newborn screening experienced physicians. This may also reflect a lack of awareness of genetic counseling as a health service. Medical centers providing genetic counseling services may need to do more marketing to increase their visibility in the community.

One of the nonreferring physicians who is outside of the metro area reported having no genetic counselor in the area, therefore reinforcing the need for genetic counseling services and specialists in nonmetro areas. One physician's comments reflect this need: "If we expand the screening for more diseases, we will need more support and options for referral." This barrier to new- 
born blood spot screening has been identified in the past, and outreach clinics may be able to address the need for genetic services in nonmetro areas. ${ }^{10}$ Also, physicians may consider referring patients to genetic counseling, despite the distance. Patients have reported improved communication with their partners and other family members after genetic counseling. ${ }^{18}$ Also, patients who receive genetic counseling have a significantly greater understanding of genetic implications. ${ }^{9}$

The physicians who had referred patients to genetic counseling were overwhelmingly satisfied with their services (99\%). Only one physician was not satisfied because genetic counselors were not easily available, although (s)he did appreciate all of the proposed benefits of genetic services. This lack of availability may point to an increased need for genetic counseling services in general. Physician satisfaction with genetic counseling, regardless of the reason for referral, also may affirm the overall efficiency and effectiveness of genetic counseling. Based on physician response, specific concerns about current methods used in genetic counseling were not raised.

This survey has a number of strengths. The results reflect a population experienced with babies who have had an abnormal newborn blood spot screening result, although they were not selected for this characteristic. The majority of physicians had one to three babies with an abnormal newborn blood spot screening result in the last 5 years. Also, $87 \%$ of physicians had referred to genetic services. Based on such experience, this population is likely to have a valuable perspective on information they would need in caring for a baby with an abnormal newborn blood spot screening result, along with their critique of genetic services. Also, the average experience of primary physicians in this study population, 13.7 years, indicates that they may know what information they need to provide optimal health care for their patients. These physicians would potentially be familiar with genetics and general newborn blood spot screening if this was included in their training programs or medical school curriculum. However, recent reports recognize that genetics in clinical teaching is not well integrated into medical school education. In addition, findings of this study do not appear to be skewed toward one population (physician location, specialty, or years of experience). There was an equal representation of metro and nonmetro physicians $(51 \%$ and $48 \%$, respectively), which is representative of the population of Minnesota; $51 \%$ of people live in the metro area and $49 \%$ live in the nonmetro area. ${ }^{22}$

A number of limitations were present in this survey. Although the return rate of $28 \%$ is typical of the physician population in Minnesota and nationally, a sample involving 80 physicians may be biased. They may not represent all physicians who treat newborns, and included some physicians who do minimal newborn care. An attempt was made to analyze smaller groups to look for significant differences between them. However, some groups were so small that their preferences cannot translate to a larger population. As in any survey, there may be a hidden bias between responders and nonresponders reflecting experience with newborn blood spot screening or satisfaction with genetic services. Also, the word- ing of some of the questions may have been confusing or vague to physicians. Inclusion of more answer choices may have better assessed physicians' actual awareness. Another limitation is the recall of past events by physicians, which may not be accurate. Conclusions based on these results should be used with discretion. These limitations should be taken into consideration when creating any educational materials, or expanding genetic services.

Parents may have suggestions for primary care physicians or other health care professionals in helping patients identified by newborn blood spot screening. In a previous study of parents with children with hemoglobinopathies, experiences of parents suggested that the education from their primary physicians was insufficient. ${ }^{8}$ When parents of children identified as cystic fibrosis (CF) carriers were surveyed, they recommended "physicians be better informed of the details and implications of positive screening results for CF." 9 Future studies in the context of expanded screening may help determine recommendations specific to disorders included in newborn blood spot screening with MS/MS technology.

The purposes of this study were to gather the primary care providers' perspective on information and format for newborn blood spot screening quick reference material, and assess their knowledge and satisfaction with genetic services and genetic counseling. The results suggest that physicians are interested in receiving additional material, and are satisfied with the genetic counseling their patients have received. The majority of physicians prefer a laminated information sheet with the practitioner procedure for abnormal newborn blood spot screening results; the newborn screening program protocol for abnormal results, a list of diseases screened for in newborn blood spot screening, and the disease treatment options and follow-up. While an overwhelming majority of the physicians were satisfied with genetic counseling, only half agreed with specified potential benefits of genetic services. Future public health efforts by newborn screening programs or genetics clinics that include education regarding benefits of genetic services may increase physician awareness and subsequent medical benefits for people with genetic diseases. These findings will assist future efforts of physician education and expansion of genetic services due to the incorporation of MS/MS technology in newborn blood spot screening.

\section{ACKNOWLEDGMENTS}

The authors thank the following people for their advice, time, and assistance in the completion of this project: Carolyn Anderson, RN, PNP, MSPH; Dianne Bartels, RN, MA, PhD; Mark McCann, MS; Nancy Vanderburg, BSN; and the staff of The Statistical Clinic at The University of Minnesota, Twin Cities.

\section{References}

1. Desposito F, Lloyd-Puryear MA, Tonniges TF, Rhein F, Mann M. Survey of pediatrician practices in retrieving statewide authorized newborn screening results. Pediatrics 2001;108:E22.

2. American Academy of Pediatrics Newborn Screening Task Force. Serving the family from birth to the medical home: newborn screening. A blueprint for the future: a call 


\section{Thompson et al.}

for a national agenda on state newborn screening programs. Pediatrics 2000;106: $389-427$.

3. Minnesota Department of Health. http://www.health.state.mn.us.divs/fh/mcshn/ nbshome.htm. Minnesota Newborn Blood Spot Screening Accessed May 31, 2005.

4. Centers for Disease Control and Prevention. Using tandem mass spectrometry for metabolic disease screening among newborns: a report of a work group. Morb Mortal Wkly Rep April 13, 2001;50:1-34.

5. Lloyd-Puryear MA, Forsman I. Newborn screening and genetic testing. J Obstet Gynecol Neonatal Nurs 2002;31:200-207.

6. Korson MS. Advances in newborn screening for metabolic disorders: what the pediatrician needs to know. Pediatr Ann 2000;29:294-301.

7. Kim S, Lloyd-Puryear MA, Tonniges TF. Examination of the communication practices between state newborn screening programs and the medical home. Pediatrics 2003;111:e120-e126.

8. Steinberg Warren N, Carter TP, Humbert JR, Rowley PT. Newborn screening for hemoglobinopathies in New York State: experience of physicians and parents of affected children. J Pediatr 1982;100:373-377.

9. Ciske DJ, Haavisto A, Laxova A, Rock LZM, Farrell PM. Genetic counseling and neonatal screening for Cystic Fibrosis: an assessment of the communication process. Pediatrics 2001;107:699-705.

10. U.S. Department of Health and Human Services, Health Resources and Services Administration, Maternal and Child Health Bureau Newborn Screening: Toward a Uniform Screening Panel and System Report for Public Comment http://www. mchb.hrsa.gov/screening. Accessed May 31, 2005.

11. American College of Medical Genetics/American Society of Hum Genet Test and Technology Transfer Committee Working Group. Tandem mass spectrometry in newborn screening. Genet Med 2000; 2:267-269.
12. Bleiker, EM, Aaronson NK, Menko FH, Hahn DE, et al. Genetic counseling for hereditary cancer: a pilot study on experiences of patients and family members. Patient Educ Couns. 1997;32:107-116.

13. Berkenstadt M, Shiloh S, Barkai G, Katzne1son MBM, Goldman B. Perceived personal control (PPe): a new concept in measuring outcome of genetic counseling. Am J Med Genet 1999;82:53-59.

14. Stockdill SH. How to evaluate foundation programs. St, Paul: St Paul Foundation, 1998.

15. The R Development Core Team 2003. [web based free software]. Version 1.6.2. http://rweb.statumn.edu/cgi-bin/Rweb/Rweb.cgi. Accessed May 31, 2005.

16. Wheeler PG, Smith R, Dorkin H, Parad RB, Comeau AM, Bianchi DW. Genetic counseling after implementation of statewide cystic fibrosis newborn screening: two years' experience in one medical center. Genet Med 2001;3:411-415.

17. Farrell MH, Certain LK, Farrell PM. Genetic counseling and risk communication ser vices of newborn screening programs. Arch Pediatr Adolesc Med 2001;155:120-126.

18. Bernhardt BA, Biesecker BB, Mastromarino CL. Goals, benefits, and outcomes of genetic counseling: client and genetic counselor assessment. Am J Med Genet 2000; 94:189-197.

19. National Society of Genetic Counselors, 1983. Available at: http://www.nsgc. org!about!definition.asp. Accessed May 31, 2005.

20. Kaye CI, Laxova R, Livingston JE, Lloyd-Puryear MA, et al Integrating genetic services into public health- guidance for state and territorial programs from the National Newborn Screening and Genetics Resource Center (NNSGRC). Community Genet 2001;4:175-196.

21. Korf BR. Integration of genetics into clinical teaching in medical school education Genet Med 2002 4, Supplement:33S-38S. Newborn blood spot screening

22. Reddy H, Harris I, Galle B, Seaquist ER. Continuing medical education: what do Minnesota physicians want? Minn Med 2001;84:58-61. 